\title{
A prospective study on management of polytrauma with early appropriate care
}

\author{
Sandeep DN ${ }^{1}$, Vaibhav Bhadbhade ${ }^{2 *}$
}

${ }^{1}$ Registrar, ${ }^{2}$ Assistant Professor, Dept. of Orthopaedics, ${ }^{1}$ Sagar Hospitals, Bangalore, Karnataka, ${ }^{2}$ Srinivas Institute of Medical Sciences and Research Centre, Mangalore, Karnataka, India

*Corresponding Author: Vaibhav Bhadbhade

Email: vaibhav_rb9@hotmail.com

\begin{abstract}
Introduction: The definition of polytrauma varies among different specialty and different countries, polytrauma patients are the subgroup of severely injured patients who have sustained injuries to more than one body region and organ with any one of the injury being life threatening. Early appropriate care (EAC), is an immediate resuscitative treatment protocol in orthopaedic trauma management in which we aim to feature any major trauma and address the most time critical injuries of body without adding to their physiological overload to the system. The aim of early appropriate care is to fix and stabilize the central skeletal system along with peripheral long bones of the skeletal system to reduce the risk of complications.

Materials and Methods: It is a prospective observational study conducted from October 2016 to November 2017 and 29 patients in the study with a mean age of 35years. All patients were managed by Early Appropriate Care. The average time taken to resuscitate the patients was $8 \mathrm{hrs}$. Following surgery patients were followed up for one month to observe post operative complications.

Results: In our series 3 patients developed pulmonary embolism, patients who developed complications were managed successfully without any mortality. Thus, a low incidence of mortality seems to be associated with EAC protocol in our series as well as in the existing literature.

Conclusion: Following adequate resuscitation and correction of acidosis early definitive fixation is possible for bony injuries of polytrauma patients which avoids the need of second surgery, also reduces the hospital stay duration, reduces post operative complications and early rehabilitation protocols can be made.
\end{abstract}

Keywords: Early appropriate care, EAC, Polytrauma management.

\section{Introduction}

Polytrauma is a major cause of death and disability worldwide mainly effects young adults and elderly population. ${ }^{1}$

The definition of polytrauma varies among different specialty and different countries, polytrauma patients are the subgroup of severely injured patients who have sustained injuries to more than one body region and organ with any one of the injury being life threatening. Polytrauma is a syndrome of multiple injuries exceeding a defined severity (ISS $\geq 17$ ) with sequential systemic reactions that may lead to dysfunction or failure of remote organs and vital systems, which have not themselves been directly injured. ${ }^{2,3}$

Polytrauma induces massive physiological changes by alteration of metabolic pathway and activation of innate immune system. The post traumatic metabolic changes includes hypermetabolism with increased energy expenditure, increased protein catabolism, hyperglycemia associated with insulin resistance and metabolic acidosis with hyperlactatemia..$^{4-8}$

$90 \%$ of polytrauma patients will have a bony injury, orthopaedics accounts for $50 \%$ of the operation undertaken, And timing of surgical intervention for these patients remain controversial. $^{9}$

'Early total care', the first definitive orthopaedic management of all bony fractures is difficult since the additional hemorrhage with orthopaedic surgery may sometimes be connected with a deleterious systemic inflammatory response(SIRS) and related complications in under resuscitated patients, generating a 'second hit' beyond the systemic impact of the injury itself (first hit).
In 2000, Scalea et $\mathrm{al}^{9}$ coined the term 'Damage Control Orthopaedics'-(DCO), the aim was to prevent exsanguinations and death, rather than to definitely treat the broken bone. Stabilization of patient rather than fixation of broken bone becomes important in case of polytrauma, hence the purpose of Damage Control Orthopaedics is to prevent the patients condition worsen by the 'second hit' of a major orthopaedic surgical procedure and to wait for the definitive surgical fracture fixation upto a time where patient is medically fit to undergo the proposed orthopaedics surgery without any major grave risks. Minimally invasive surgical techniques such as external fixation are used initially. Then definitive surgeries will be done in later days.

Early appropriate care (EAC), is an immediate resuscitative treatment protocol in orthopaedic trauma management in which we aim to feature any major trauma and address the most time critical injuries of body without adding to their physiological overload to the system.

The aim of early appropriate care is to fix and stabilize the central skeletal system along with peripheral long bones of the skeletal system to reduce the risk of complications. These patients benefit only with adequate resuscitation and haemodynamic stabilization along with early skeletal injury fixation.

Polytrauma cases with mechanically unstable fractures of the central skeletal bones like pelvis, acetabulum and spine along with longer bones like femur will be stabilized definitively within first 36 hours in patients who responded to a positive response to early and adequate resuscitative care, defined as having either a lactate $<4.0 \mathrm{mmol} / \mathrm{L}$, PH $\geq 7.25$,or base excess $(\mathrm{BE}) \geq-5.5 \mathrm{mmol} / \mathrm{L}$. So it is very 
important to resuscitate the polytrauma patients and to acquire normal laboratory parameters before surgery to reduce post-operative complications. ${ }^{11-13}$

\section{Materials and Methods}

It is a prospective observational study done with 29 patients with polytrauma were included in the study all were managed by Early Appropriate Care. Polytrauma was the result of road traffic accidents in all patients. The duration of the study was between October 2016 and November 2017. There were 29 patients out of which 25 were males and 4 patients were females. The mean age of the patient was 35yrs (range: 19yrs-52yrs). The inclusion and exclusion criteria to include polytrauma patients in study as listed below.

\section{Inclusion Criteria}

1. Adults patients with polytrauma

2. Patients with bilateral femoral fractures

3. ISS score $>16$

\section{Exclusion Criteria}

1. Patients were excluded for having injuries sustained from low energy mechanisms

2. Skeletally immature patients

3. Having an ISS score $<16$

4. Patients with definitive non operative treatment

5. Patients reporting to casualty after 24 hours of incidence

6. Referring from other hospital following primary resuscitation

7. Patients who needs immediate surgeries

All polytrauma patients getting admitted from the casualty were initially evaluated and assessed, proper history of the injury elicited, rapid systematic assessment is performed immediately to identify the possible life threatening conditions, fracture characteristics, associated injuries, medical comorbidities, and the timing and techniques of provisional treatment were documented. Vital signs and laboratory parameters were documented. On arrival to casualty blood investigations for total count, haematocrit, blood grouping, arterial blood gas values, lactic acid, ph and base excess were obtained. Once the patients vitals are stabilized secondary survey has been done this being a complete acute diagnostic checkup. Various organ systems involved in trauma were assessed and ISS scoring system was applied to grade the severity of polytrauma. Injury Severity Score (ISS) $>16$ were involved in the study.

Following initial assessment, stabilization and trauma scoring patients were shifted to ICU for further management, further evaluation of the patient done in ICU to obtain other associated medical conditions like hypertension, diabetes and previous cardiac respiratory problems, depending on the severity of injury and acidosis, patients managed aggressively by giving crystalloids, colloids and sodium bicarbonates, blood in case of patients with severe blood loss and in haemorrhagic shock. The second sample to asses haematocrit values, $\mathrm{Hb}$, arterial blood gas values, lactic acid, ph and base excess was sent 8 hours following admission preoperatively following correction of acidosis and blood loss.

Once the acidosis is corrected which is assessed by ph, lactic acid and base excess values, patients were taken up for definitive surgeries according to fracture pattern and type of anaesthesia was chosen accordingly, we could neutralize the acidity level of all patients included in the study within 812 hours, Following surgery patients shifted to ICU for post operative care, proper post operative care was done by ICU intensivist and the third sample 12 hours following surgery for ph base excess and lactic acid obtained. Once these values are normalized patients shifted out from ICU.

Total hospitalization duration, length of ICU stay required with or without ventilator assistance required and also the transfusion requirements during the whole course of treatment were determined. Complications associated to polytrauma and its treatment were assessed, like local wound infections and sepsis if associated, head injuries, pulmonary complications like adult respiratory distress syndrome; pulmonary embolism; pneumonia due to longer ICU stay, acute renal failure, multiple organ dysfunction syndrome (MODS), and deep venous thrombosis (DVT).

Patients were followed up for one month to assess the above mentioned complications. Out of 29 patients, one patient needed ventilator support who developed pulmonary complications postoperatively.

\section{Results}

\section{Characteristics of Cases Studied}

Table 1: Age distribution

\begin{tabular}{|l|c|c|}
\hline \multirow{2}{*}{ Age group } & \multicolumn{2}{|c|}{ Cases } \\
\cline { 2 - 3 } & No. & \% \\
\hline Up to 20years & 3 & 10.3 \\
\hline $21-30 \mathrm{yrs}$ & 6 & 20.7 \\
\hline $31-40 \mathrm{yrs}$ & 12 & 41.4 \\
\hline Above 40yrs & 8 & 27.6 \\
\hline Total & 29 & 100.0 \\
\hline Range & & $19-52 \mathrm{yrs}$ \\
& & $35.0 \mathrm{yrs}$ \\
Mean S.D. & & $9.5 \mathrm{yrs}$ \\
\hline
\end{tabular}

Table 2: Injury severity score

\begin{tabular}{|l|c|r|}
\hline \multirow{2}{*}{$\begin{array}{c}\text { Injury Severity } \\
\text { Score }\end{array}$} & \multicolumn{2}{|c|}{ Cases } \\
\cline { 2 - 3 } Up to 20 & No. & \% \\
\hline $\mathbf{2 1 - 3 0}$ & 1 & 3.5 \\
\hline $\mathbf{3 1 - 4 0}$ & 9 & 31.0 \\
\hline $\mathbf{4 1 - 5 0}$ & 9 & 31.0 \\
\hline Above 50 & 7 & 24.1 \\
\hline Total & 29 & 10.3 \\
\hline Range & & 100.0 \\
Mean S.D. & & $19-66$ \\
& & 36.97 \\
\end{tabular}


Table 3: Post operative complications

\begin{tabular}{|l|c|c|}
\hline \multirow{2}{*}{ Post operative Complications } & \multicolumn{2}{|c|}{ Cases } \\
\cline { 2 - 3 } & No. & \% \\
\hline Present (Pulmonary Embolism) & 3 & 10.3 \\
\hline Absent & 26 & 89.7 \\
\hline Total & 29 & 100.0 \\
\hline
\end{tabular}

Table 4: Changes in lactic acid values

\begin{tabular}{|l|c|c|c|}
\hline \multirow{2}{*}{ Lactic Acid Value } & \multicolumn{3}{|c|}{ Lactic Acid Value } \\
\cline { 2 - 4 } & Range & Mean & S.D. \\
\hline Initial & $4-8$ & 5.61 & 0.98 \\
\hline $\begin{array}{l}\text { 8 hours after } \\
\text { stabilization } \\
\text { (preoperative) }\end{array}$ & $0.7-4.4$ & 2.24 & 1.01 \\
\hline 12 hours after surgery & $0-1.9$ & 0.69 & 0.52 \\
\hline Change in 8 hours & $-5.0-(-2.6)$ & -3.37 & 0.57 \\
\hline $\begin{array}{l}\text { Change in 12 hours } \\
\text { after surgery }\end{array}$ & $-6.7-(-3.6)$ & -4.92 & 0.77 \\
\hline
\end{tabular}

Table 5: Changes in ph levels

\begin{tabular}{|l|c|c|c|}
\hline \multirow{2}{*}{ PH levels at } & \multicolumn{3}{|c|}{ PH levels } \\
\cline { 2 - 4 } & Range & Mean & S.D. \\
\hline Initial & $7.18-7.4$ & 7.3 & 0.06 \\
\hline $\begin{array}{l}\text { 8 hours after } \\
\text { stabilization }\end{array}$ & $7.24-7.46$ & 7.37 & 0.06 \\
\hline $\begin{array}{l}12 \text { hours after } \\
\text { surgery }\end{array}$ & $7.28-7.46$ & 7.4 & 0.05 \\
\hline $\begin{array}{l}\text { Change in 8 } \\
\text { hours }\end{array}$ & $0.01-0.2$ & 0.07 & 0.05 \\
\hline $\begin{array}{l}\text { Change in 12 } \\
\text { hours after } \\
\text { surgery }\end{array}$ & $0.02-0.2$ & 0.1 & 0.05 \\
\hline
\end{tabular}

Table 5: Changes in base excess values

\begin{tabular}{|l|c|c|c|}
\hline Base Excess values at & \multicolumn{3}{|c|}{ Base Excess values } \\
\cline { 2 - 4 } & Range & Mean & S.D. \\
\hline Initial & $-10.1-(+1.0)$ & -3.45 & 2.71 \\
\hline $\begin{array}{l}\text { 8 hours after } \\
\text { stabilization }\end{array}$ & $-4-(+1.0)$ & -1.75 & 1.12 \\
\hline 12 hours after surgery & $-4-(+1.0)$ & -0.27 & 1.38 \\
\hline Change in 8 hours & $-1.8-(+6.1)$ & 1.7 & 2.11 \\
\hline $\begin{array}{l}\text { Change in 12 hours } \\
\text { after surgery }\end{array}$ & $-0.3-(+8.7)$ & 3.18 & 2.05 \\
\hline
\end{tabular}

\section{Discussion}

Debate still persists on the decision for Damage Control Orthopaedics (DCO) over early total care (ETC) of the patients. Clinically and mechanically unstable fractures of the long bones, and central skeletal system like pelvis, acetabulum, and thoracic- lumbar spine often require complete immobilization and bedrest until surgery. Damage control orthopaedics offers temporary stabilization to limit the complications, while providing time for complete recovery of the body. This demands in additional surgical interventions with require mechanical stabilizers like orthopaedic implants and splints increasing the hospital tenure. Fracture patterns of those of the acetabulum-pelvis complex, and central spine complex (cervical spine, thoracic spine or lumbar spine) may prevent Damage Control Orthopaedics and, even if temporary stabilization is feasible, may not allow early mobilization of the patient. There are likely favourable conditions and possibilities along the management spectrum from Early Total Care to Damage Control Orthopaedics when early total care is adequate and appropriate which may be undertaken.

Major polytrauma cases many times requires and demands profound resuscitative measures to correct deranged blood markers and volume losses in shock and the resulting derangement of metabolic acidosis, reflected by changes in $\mathrm{pH}$ and excess base. These conditions demands quick transfusions of crystalloid, colloid, packed red blood cells (PRBC), fresh frozen plasma (FFP), and platelets (Plt) to maintain the haemostasis. Metabolic acidosis on presentation is a prognostic indicator for the development of pulmonary complications. ${ }^{15-17}$ organ dysfunction, and death. ${ }^{17-20}$

The EAC protocol relies on continuous reassessment of acidosis as response to resuscitation, Surgery is permitted when specific laboratory criteria are met, and definitive fixation occurs within 36 hours of injury. The protocol will reduce pulmonary and other complications and will also reduce length of hospital stay. ${ }^{11-13,15}$ Hence it is presumed that adherence to the early appropriate care-EAC protocol will be beneficial to the patient in all aspects by mainly reducing hospital stay due to adequate management of early complications and in turn aiding the financial benefits to the patients.

Definitive orthopaedic management protocol for clinically and mechanically unstable fractures of the long bones and central skeletal system bones like pelviacetabular complex, and dorso-lumbar spine was carried out within 36 hours in patients who responded adequately and positively to early resuscitative efforts, defined as having either a lactate $<4.0 \mathrm{mmol} / \mathrm{L}, \mathrm{pH} \geq 7.26$, or base excess (BE) $\geq-5.5 \mathrm{mmol} / \mathrm{L}$. Then post operatively complications are measured as outcome of resuscitation. Complications associated with the injury like the local wound infections and sepsis if associated, head injuries, pulmonary complications like adult respiratory distress syndrome; pulmonary embolism; pneumonia due to longer ICU stay, acute renal failure, multiple organ dysfunction syndrome (MODS), and deep venous thrombosis (DVT).

In our study, a total of 29 patients were managed according to the EAC protocol. The serum lactate, $\mathrm{pH}$ and base excess were assessed in all the patients on arrival at the casualty department. This was followed by resuscitation of the patients to optimize the haemodynamic parameters. The metabolic markers (lactate, $\mathrm{pH}$ and base excess) were reassessed after 8 hours of resuscitation. If these parameters had returned to normal, the patients were shifted to the operation theatre for definitive stabilization of the bony injuries. If the parameters had not returned to normal levels by 8 hours, resuscitation was continued till the parameters returned to normal. In the earlier literature, resuscitation was continued till 12 to 15 hours to normalize the parameters. ${ }^{15}$ 
In our study, a maximum of 12 hours of resuscitation was required in only 2 of the 29 patients. Thus definitive operations are not unduly delayed by the resuscitation protocol.

In our series of 29 patients managed using EAC protocol, three patients had major complications. Two out of three cases developed fat embolism following surgical intervention as there was involvement of long bones and one patient developed deep vein thrombosis (DVT) and ultimately leading to pulmonary embolism (PE). All these cases were treated and managed in the intensive care unit (ICU) for an average period of around 1 week. One patient with DVT and PE required mechanical ventilation whereas in the other two patients with fat embolism, oxygenation via mask, intravenous fluids and supportive care were sufficient. The patient with deep vein thrombosis was treated using low molecular weight heparin (LMWH) injections. The complication rates in our series are comparable with those reported earlier by Vallier HA et al $2013^{11}$ and Vallier HA et al $2015^{12}$ which is $12 \%$ and $16.3 \%$ respectively. Even those patients who developed complications were managed successfully without any mortality. Thus, a low incidence of mortality seems to be associated with EAC protocol in our series as well as in the existing literature ${ }^{11,12,15}$ by reducing the complication rates and shorter ICU and hospital length of stay.

All the patients in our case series spent an average duration of 12.7 days in hospital. This is comparable to the average hospital stay duration in the existing literature by Vallier HA et al 2013, ${ }^{11}$ Vallier HA et al $2015^{12}$ and Nahm J et al $2011^{15}$ which is $10.8,9.52$ and 11.2 days respectively. This reduces the economic burden on the patients and the risk of nosocomial infections. The requirement for repeated secondary surgical interventions are also very low in the EAC protocol. There were no patients in our series who needed secondary surgical procedures prior to discharge from the hospital. This is the experience of other authors also who have adopted the EAC protocol. ${ }^{11,12}$ Absence of repeated surgical interventions reduces the morbidity and allows rapid rehabilitation of these patients.

In our study, patients with poly trauma were commoner in the 31-40 age groups with average age being 35 years. Mode of injury in all patients was road traffic accidents. This could be attributed to increased mobility in urban working people. $2^{\text {nd }}$ peak was $>40$ years age groups. Our study findings are comparable to the study made by Nahm $\mathbf{J}$ et al 2011. ${ }^{15}$

In our study there were three patients who developed pulmonary embolism, two out of them were younger age group patients $(<30 y)$ and the remaining was older age group patient $(>45 y)$ which is statistically not significant. Our study is comparable with the Reich et al $(2016){ }^{14}$ which concludes that EAC is associated with comparable complication rates in young and elderly patients. Our series had a male predominance with $86.2 \%$ and $13.8 \%$ females which is comparable with all other studies. Male predominance can be well explained due to increased outdoor activity of males.
In our series there were four female patients of whom two patients developed pulmonary embolism. The relation between sex distribution and postoperative complications has not yet been reported in the literature. This complications may be attributed to higher ISS score and higher ASA grade in the two female patients in our series. Further studies involving larger cohorts of patients are essential to determine if gender differences exist in the response of patients to the EAC protocol.

In our studies we have included the patients with ISS score more than 16. Injury severity score (ISS) is an anatomical scoring system that provides an overall score for patients with multiple injuries. It was understood that there was a statistically significant association between postoperative complications and ISS. Three complications were seen in cases with mean ISS score of 55.0 and no complications were seen in cases with mean ISS score of upto 34.88. Our study is comparable with other studies stating that ISS grading is a reliable predictor of postoperative complications, mortality and morbidity. It is correlated to the length of hospital stay ie patients with longer hospital stay and a higher complication rates had higher ISS scores.

\section{Conclusion}

Following adequate resuscitation and correction of acidosis early definitive fixation is possible for bony injuries of polytrauma patients which avoids the need of second surgery, also reduces the hospital stay duration, reduces post operative complications and early rehabilitation protocols can be made. Within the first 8 hours, correction of lactate, $<4.0 \mathrm{mmol} / \mathrm{L}, \mathrm{pH} .>7.25$, or $\mathrm{BE}$ less than $5.5 \mathrm{mmol} / \mathrm{L}$ is associated with a reduced risk of pulmonary complications. Presence and severity of chest injury, head injury, number of fractures to be treated, and timing of fixation are other significant variables to include in a predictive model for complications. So we conclude that by adequate resuscitation of polytrauma patients, they can be taken up for early definitive fixation and can expect minimal postoperative complications and also reduce the hospital length of stay This should translate in to lower costs of care.

\section{Conflict of Interest: None.}

\section{References}

1. World Health Organisation (WHO). World health statistics. Retrieves April 15,2009, from http://www.who.int/whosis/en

2. American college of surgens/committee of trauma. National trauma data bank annual report 2005, dataset version 5.0 Chicago: American college of surgoens ontrauma 2005.

3. Sikand M, Williams K, White C, Moran CG. The financial cost of treating polytrauma: implications for tertiary referral centres in the United Kingdom. Inj 2005;36:733-7.

4. Plank LD, Hill GL. Energy balance in critical illness. Proc Nutr Soc 2003;62:545-52.

5. Griffiths RD, Hinds CJ, Little RA. Manipulating the metabolic response to injury. Br Med Bull 1999;55:181-95.

6. Keel M, Trentz O. Pathophysiology of polytrauma. Inj 2005;36:691-709. 
7. Schlag G, Redl H. Mediators of injury and inflammation. World J Surg 1996;20:406-10.

8. Redl H, Bahrami S, Fürst W, Schiesser A, Schlag G, Mollnes TE. Evidence for early bacteremia/endotoxemia and inflammatory responses after trauma: hemorrhage and complement activation in a nonhuman primate model. Eur J Trauma 2000;26:300-7.

9. Scalea TM. Optimal timing of fracture fixation: have we learned anything in the past 20 years? J Trauma 2008;65:25360

10. Riska EB, Myllynen P. Fat embolism in patients with multiple injuries. J Trauma 1982;22:891-4

11. Vallier HA, Wang X, Moore TA, Wilber JH, Como JJ. Timing of orthopaedic surgery in multiple trauma patients: development of a protocol for early appropriate care. J Orthop Trauma 2013;27(10):543-51.

12. Vallier HA, Moore TA, Como JJ, Wilczewski PA, Steinmetz MP, Wagner KG, et al. Complications are reduced with a protocol to standardize timing of fixation based on response to resuscitation. J Orthop Surg Res 2015;10:155.

13. Weinberg DS, Narayanan AS, Moore TA, Vallier HA. Prolonged resuscitation of metabolic acidosis after trauma is associated with more complications. J Orthop Surg Res 2015; 10:153.

14. Reich MS, A. J. Dolenc, T. A. Moore and H. A. Vallier. Is Early Appropriate Care of axial and femoral fractures appropriate in multiply injured elderly trauma patients? $J$ Orthop Surg Res 2016;11:106.

15. Nahm NJ, BS, Como JJ, Wilber JH, Vallier HA. Early Appropriate Care: Definitive Stabilization of Femoral Fractures within 24 Hours of Injury Is Safe in Most Patients with Multiple Injuries. J Trauma 2011;71:175-85.

16. Eberhard LW, Morabito DJ, Matthay MA, Mackersie RC, Campbell AR, Marks JD,et al. Initial severity of metabolic acidosis predicts the development of acute lung injury in severely traumatized patients. Crit Care Med 2000;28(1):12531.

17. Davis JW, Parks SN, Kaups KL, Gladen HE, O’Donnell-Nicol S. Admission base deficit predicts transfusion requirements and risk of complications. J Trauma 1996;41(5):769-74.

18. Guyette F, Suffoletto B, Castillo JL, Quintero J, Callaway C, Puyana JC. Prehospital serum lactate as a predictor of outcomes in trauma patients: a retrospective observational study. J Trauma 2011;70(4):782-6.

19. Callaway DW, Shapiro NI, Donnino MW, Baker C, Rosen CL. Serum lactate and base deficit as predictors of mortality in normotensive elderly blunt trauma patients. J Trauma 2009;66(4):1040-4.

20. Claridge JA, Crabtree TD, Pelletier SJ, Butler K, Sawyer RG, Young JS. Persistent occult hypoperfusion is associated with a significant increase in infection rate and mortality in major trauma patients. J Trauma 2000;48(1):8-14.

How to cite this article: Sandeep DN, Bhadbhade V. A prospective study on management of polytrauma with early appropriate care. Indian $J$ Orthop Surg 2019;5(2):118-22. 